\title{
Theranostics: Cancer imaging and therapy using injectable radionuclide-labeled ligands
}

\begin{abstract}
Cytotoxic chemotherapy, a mainstay of non-surgical cancer therapy for many decades and often combined with external beam radiotherapy, can be viewed as a "carpet bombing" therapeutic approach, often resulting in substantial side effects and recurring cancer cells. In the last decade, a new molecular oncology model has evolved that includes genomics, proteomics and immune system modulation. Targeted therapies and immunotherapies have offered new, albeit more expensive, therapeutic modalities. However, these typically benefit only a subset of patients with immunohistochemistry-identified markers (e.g., CTLA-4 or PD-L1) or specific gene mutations (e.g., EGFR, ALK, BRAF, PIK3CA) and tend to suppress rather than eradicate tumors. Targeted precision cancer therapy using injectable radionuclide-labeled ligands has opened up new horizons to treat resistant or widely scattered cancers by delivering cytotoxic ionizing radiation directly and specifically to tumor cells. This therapeutic approach is termed radioligand theranostics (RT), a combination of therapeutics + diagnostics. This two-step process first uses molecular imaging to identify a specific receptor or biomarker expressed on a particular tumor cell type and then uses a therapeutic version of the radioligand that is internalized by cancer cells via specific receptors to achieve cell death with minimal damage to neighboring healthy tissue. One of the most successful examples of RT development includes octreotide radionuclide scintigraphy and therapy for neuroendocrine tumors (NETS). ${ }^{68} \mathrm{Ga}$ DOTATOC (DOTA-Phe-Tyr octreotide) injection is now available for PET imaging of somatostatinpositive gastroenteropancreatic NETs and ${ }^{177} \mathrm{Lu}$-DOTA-Tyr ${ }^{3}$-octreotate was approved by both the FDA and EMA to treat patients with progressive somatostatin-receptor-positive midgut NETs. However, RT has widespread therapeutic implications beyond NETS and has served as a beacon for the conception of theranostic treatments for other cancer types, for example multiple myeloma, leukemia, non-Hodgkin lymphoma, metastatic castrateresistant prostate cancer, ovarian cancer, and breast cancer.
\end{abstract}

Keywords: Theranostics, imaging, radioligand, targeted radionuclide therapy, cancer, radiopharmaceuticals
Volume 8 Issue 6 - 2020

\section{Jan S Redfern}

Redfern Strategic Medical Communications, Inc., USA

Correspondence: Jan S Redfern, PhD, Redfern Strategic

Medical Communications, Inc, 700 Jay Bird Road

Springtown, Texas 76082, United States of America

Email jan@redfernstrategic.com

Received: October 20, 2020 | Published: November 16, 2020
Abbreviations: Ag, tumor antigen; AKT, serine-threonine kinase; $\mathrm{CD}$, cluster of differentiation; $\mathrm{CT}$, computed tomography; CTLA-4, cytotoxic T-lymphocyte-associated protein 4; CXCR4, C-X-C chemokine receptor type 4; DNA, deoxyribose nucleic acid; DOTA, 1,4,7,10-tetraaza-cyclododecane-tetraacetic acid; DTPA, diethylene triamine pentaacetic acid; DOTATOC, (DOTA-Phe-Tyr)octreotide; DOTADATE, DOTA-0-Tyr ${ }^{3}$-Octreotate; EGFR, epidermal growth factor receptor; EMA, European Medicines Agency; FAPI, fibroblast activation protein inhibitor; FDA, Food and Drug Administration; FDG, fluorodeoxyglucose; GRP-R, gastrin-releasing peptide receptor; JR11, Cpa-c(dCys-Aph(Hor)-dAph(Cbm)-Lys-ThrCys)-dTyr-NH2)); mAb, monoclonal antibody; MAPK, mitogenactivated protein kinase; mCRPC, metastatic castrate-resistant prostate cancer; MHC, major histocompatibility complex; MIBG, meta-iodobenzylguanidine; mTOR, mammalian target of rapamycin; NET, neuroendocrine tumor; NETTER, Neuroendocrine Tumors Therapy; NODAGA, 1,4,7-triazacyclononane,1-glutaric acid4,7-acetic acid; p53, tumor protein; PD-1, programmed cell death protein 1; PD-L1, programmed death-ligand 1; PET/CT, positron emission tomography with computed radiographic imaging; PI3K, phosphatidylinositol-3-kinase; PRRT, peptide receptor radionuclide therapy; TCR $=$ T-cell receptor. $\mathrm{PET}$, positron emission tomography;
PSMA, prostate specific membrane antigen; RT, radioligand theranostics; SPECT, single photon emission computed tomography; TCMC, 2-(4-isothiocyanotobenzyl)-1, 4, 7, 10-tetraaza-1, 4, 7, 10-tetra-(2-carbamonyl methyl)-cyclododecane

\section{Introduction: the foundation stone of radioligand theranostics ( $R T$ )}

During part of World War 2 and in the immediate years thereafter, Dr Saul Hertz at Massachusetts General Hospital conducted his trailblazing research on the medical applications of artificial radioactivity, the process of making non-radioactive atoms radioactive by means of a particle accelerator. ${ }^{1}$ Collaborating with several physicists, Hertz used a cyclotron to generate radioactive ${ }^{130} \mathrm{I}$ and ${ }^{131} \mathrm{I}$ and subsequently utilized these nascent isotopes to diagnose and treat patients with thyroid carcinoma. In so doing, Hertz inaugurated the era of nuclear medicine and targeted precision cancer therapy and created the first Food and Drug Administration (FDA)-approved (in 1951) radiopharmaceutical for the treatment of thyroid cancer. Targeting the sodium-iodine symporter in patients with thyroid carcinoma remains a pillar of nuclear medicine to this day. ${ }^{2}$ Now, more than 80 years later, radiopharmaceutical development has taken giant leaps forward with far-reaching therapeutic implications for many other cancer 
types. This review describes the basic principle of RT, summarizes current RT modalities, and explores the future of targeted precision medicine for neuroendocrine tumors (NETs) and other tumor types.

\section{The basic principle of radioligand theranostics}

The radioligand used in theranostics comprises four component parts: ligand-linker-chelator-radioisotope (Figure 1). ${ }^{3}$ The targeting ligand seeks out and binds to the target receptor or other biomarker on the cancer cell. ${ }^{4,5}$ This ligand can be a peptide such as octreotide, a small molecule such as fibroblast-activated protein inhibitor (FAPI) or indeed an antibody against CD20, CD38, or CA45, for example. ${ }^{4}$ The ligand is also bound to a chelator (via a linker molecule), including, for example, DOTA (1,4,7,10-tetraaza-cyclododecane-tetraacetic acid) and DTPA (diethylene triamine pentaacetic acid), which are designed to complex with alpha, beta, or gamma emitters so they can be administered systemically and destroy tumor cells' DNA. The chelator "cage" can accommodate radionuclides of different energy emissions to create a theranostic pair, one for diagnosis (e.g., ${ }^{68} \mathrm{Ga}$ or $\left.{ }^{111} \mathrm{In}\right)$ and one for therapy $\left({ }^{177} \mathrm{Lu}\right.$ or $\left.{ }^{90} \mathrm{Y}\right) .^{3,6,7}$

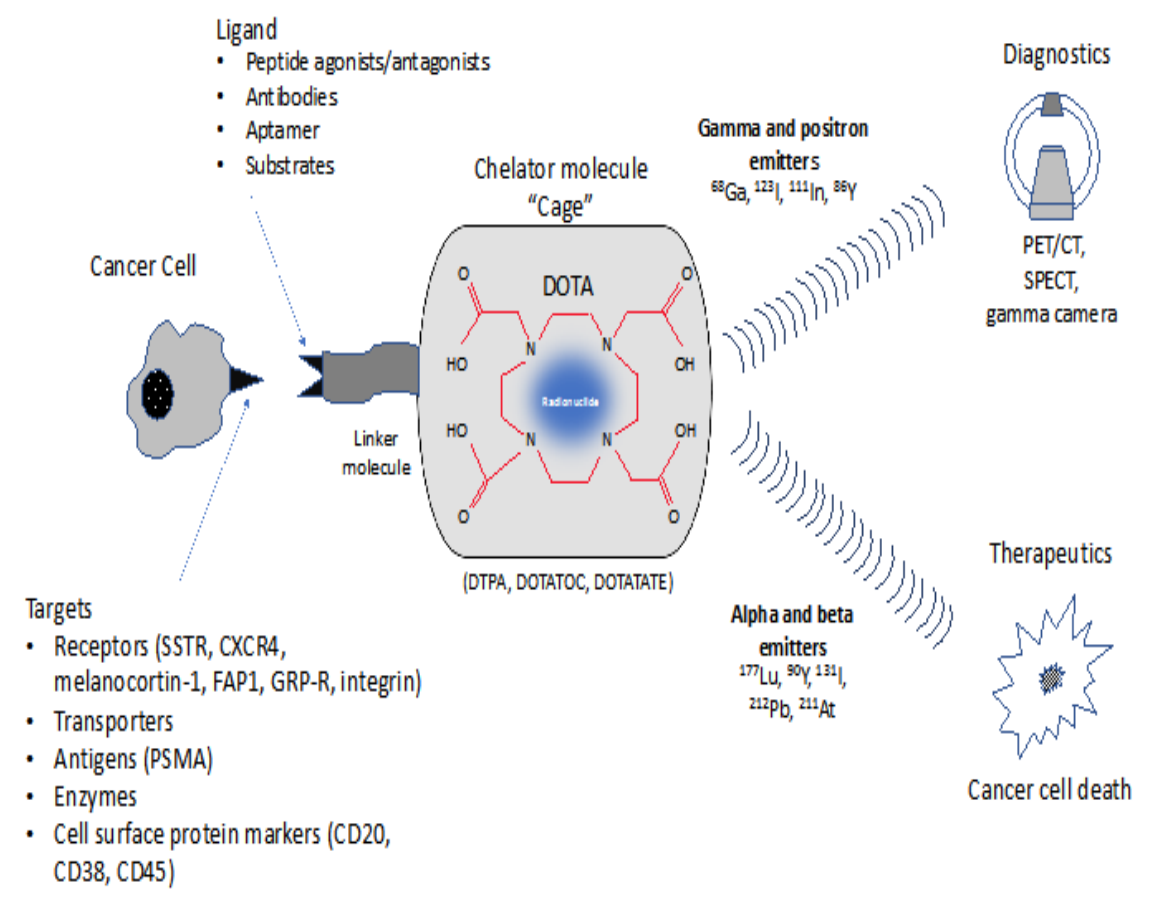

Figure I A ligand molecule such as a somatostatin analogue is conjugated to appropriate chelators (via a linker molecule) that accommodate radioisotopes for diagnostics or therapeutics.

CD, cluster of differentiation; CT, computed tomography; CXCR4, C-X-C chemokine receptor type 4; DOTA, I,4,7, I0-tetraaza-cyclododecane-tetraacetic acid; DTPA, diethylene triamine pentaacetic acid; DOTATOC, (DOTA-Phe-Tyr)-octreotide; DOTADATE, DOTA-0-Tyr ${ }^{3}$-Octreotate; FAPI, fibroblast activation protein; GRP-R, gastrin-releasing peptide receptor; PET, positron emission tomography; PSMA, prostate-specific membrane antigen; SPECT, Single Photon Emission Computed Tomography.

Although beta-emitters have typically been selected for RT, alpha emitters turn out to be more effective radioisotopes due to greater energy deposition within the cell. ${ }^{8}$ Beta emitters, such as ${ }^{177} \mathrm{Lu}$ and ${ }^{90} \mathrm{Y}$, and positron emitters (beta plus decay) such as ${ }^{68} \mathrm{Ga}$, have path lengths ranging from $\mu \mathrm{m}$ to $\mathrm{mm}$ resulting in damage to adjacent cells and normal tissues and whose energy deposition may be insufficient to cause tumor cell death. ${ }^{8}$ In contrast, alpha emitters (e.g., ${ }^{211}$ At and ${ }^{212} \mathrm{~Pb}$ ) emit densely ionizing radiation with path lengths in tissue ranging from $\mathrm{nm}$ to $\mu \mathrm{m}$ and convey high-energy radiation especially to the nucleus, with less impact on adjoining normal tissues.

\section{Radioligand theranostics for somatostatin receptor- positive NETs}

Although the general principle of RT was established in the 1940s, it took until the late 1980s before this approach gained traction and other radioligand entities were eventually developed. Early attempts in early 2000 focused on hematological malignancies (B-cell and non-Hodgkin lymphomas) that are particularly responsive to radiotherapy. ${ }^{8}$ These radio therapeutic agents targeting CD20 did not achieve widespread clinical use and one ( ${ }^{131} \mathrm{I}$-tositumomab) was voluntarily withdrawn from the market in 2013 because the postmarketing study to verify clinical benefit was not completed. ${ }^{9}$

One of the most successful examples of RT development includes octreotide radionuclide scintigraphy and therapy. In 1982, Bauer and colleagues at Sandoz (now Novartis) synthesized octreotide, a biologically stable, long-acting congener of naturally occurring somatostatin peptide..$^{10}$ Octreotide targets somatostatin receptors (primarily type 2), present on a majority of gastroenteropancreatic NETs. This congener, and others to follow (pasireotide, lanreotide), proved to be effective treatments to inhibit hormone secretion and revolutionized the treatment of gut endocrine tumors and pituitary adenomas. ${ }^{11}$ Octreotide is generally well tolerated although longterm treatment may result in biliary tract dysfunction and gallstones in some patients. ${ }^{12}$ Ongoing clinical studies using octreotide also point to its use in the treatment of other major clinical conditions as variceal bleeding, secretory diarrhea, diabetic nephropathy, carcinoid syndrome, and cancer. ${ }^{10,13}$ Octreotide's accolades don't end there-this 
congener has underpinned one of the most notable developments in the visualization, diagnosis and treatment of NETs and other tumor types. ${ }^{14}$

Krenning and colleagues at Erasmus University in Rotterdam argued that if octreotide could be radiolabeled with ${ }^{123} \mathrm{I}$ or ${ }^{131} \mathrm{I}$ (already in use in nuclear medicine), perhaps in vivo scintigraphy could similarly be utilized in humans to identify NETs that over express somatostatin receptors. ${ }^{15}$ Although methodologically challenging, Krenning eventually synthesized ${ }^{123}$ I-labeled $\mathrm{Tyr}^{3}$-octreotide and successfully used this radioligand to identify a gastrinoma, meningioma and unknown metastasis using single photon emission computed tomography (SPECT) imaging in a single patient with gastroenteropancreatic NET. ${ }^{15}$

Success of this "proof of concept" study led the investigators to continue their clinical assessment of ${ }^{123}$ I-labeled Tyr $^{3}$-octreotide as well as ${ }^{111}$ In-DPTA-D-Phe-octreotide using scintigraphy to identify known tumor locations in over 1000 patients with well-documented tumor types. ${ }^{16}$ The diagnostic accuracy of ${ }^{111}$ In-DPTA-D-Phe-octreotide in a variety of tumor types was impressive: $100 \%$ for gastrinoma, $100 \%$ for Hodgkin lymphoma, $80 \%$ for non-Hodgkin lymphoma, $96 \%$ for carcinoid and $70 \%$ for GH-producing tumor.

These early studies opened the door to a flurry of research activity using different isotopes, ligands, chelators, and formulations to optimize the diagnostic and therapeutic utility of RT agents. ${ }^{6}$ A kit $\left(\right.$ Octreoscan $\left.{ }^{\mathrm{TM}}\right)$ for the preparation of ${ }^{111}$ In-pentetreotide for imaging of the whole body and NET staging was approved by the FDA in the United States in 1994.There were delays in the licensing of therapeutic octreotide theranostic agents due to lack of objective and systematic evidence. Early studies were conducted in academic settings, in small numbers of patients, using proof-of-concept designs, and bypassed the traditional stages of drug development. ${ }^{17}$ Indeed, it has been said that the story of peptide receptor radionuclide therapy from bench to bedside perfectly illustrates how not to develop and license a new therapeutic entity. ${ }^{17}$

Eventually, however, the research was consolidated and the first randomized phase 3 trial using ${ }^{177} \mathrm{Lu}$-DOTA-Tyr ${ }^{3}$-octreotate to treat patients with progressive somatostatin-receptor-positive midgut NETs (Neuroendocrine Tumors Therapy [NETTER-1] study) was published in $2017 . .^{18}$ This study showed that addition of ${ }^{177}$ Lu-DOTATATE to octreotide depot injection resulted in $79 \%$ lower risk of disease progression or death compared with octreotide depot alone. These findings culminated in the first radiopharmaceutical for radioligand theranostics to be approved by the FDA and European Medicines Agency(EMA) ${ }^{19}$ This was followed later by FDA approval in August 2019 of ${ }^{68} \mathrm{Ga}$ DOTATOC (DOTA-Phe-Tyr octreotide) injection for PET imaging of somatostatin-positive gastroenteropancreatic NETs. ${ }^{20}$ ${ }^{68} \mathrm{Ga}$-DOTATATE positron emission tomography with computed radiographic imaging (PET/CT) has been shown to have greater accuracy for staging and superiority to Octreoscan SPECT in the detection of overall number of lesions in the body. ${ }^{21}$

Research continues in this area and PET imaging with ${ }^{68} \mathrm{Ga}-$ NODAGA (1,4,7-triazacyclononane,1-glutaric acid-4,7-acetic acid) coupled to the somatostatin receptor antagonist JR11 (Cpa-cyclo(dCys-amino-Phe-hydroorotic acid-D-4-amino-Phe(carbamoyl)-LysThr-Cys)-D-Tyr-NH2) or ${ }^{68} \mathrm{Ga}-\mathrm{OPS} 202$ is being explored along with ${ }^{177} \mathrm{Lu}-\mathrm{DOTA}-J \mathrm{R} 11$ or ${ }^{177} \mathrm{Lu}-\mathrm{OPS} 201$ as a therapeutic agent, which may transfer a greater dose of radiation to NETs even though it is not internalized within tumor cells. ${ }^{22}$ These diagnostic-theranostic pairs targeting somatostatin receptors are also being explored for possible applications to breast cancer, small cell lung cancer, renal cell cancer, and indeed, any neoplasm expressing somatostatin type 2 receptors. Further, a number of antagonist molecules, in addition to those binding to G-protein coupled receptors, are in the early stages of clinical development.

With respect to more advanced cases of NETs, expression of somatostatin receptors ebbs, and radiolabeled octreotide imaging becomes ineffectual. ${ }^{23}{ }^{18} \mathrm{~F}$ fluorodeoxyglucose $\left[{ }^{18} \mathrm{FDG}\right]$, a glucose analog taken up by metabolically active tumor cells, is extensively used in visualizing cancer lesions using PET/CT imaging. Although its usefulness in NET is limited due to the low glycolytic activity of these cancer cells, positive ${ }^{18} \mathrm{FDG}$ imaging often foreshadows a more aggressive disease course. ${ }^{24}$

\section{Protecting the kidney}

The overall safety profile for the different RTs is generally consistent, with most patients experiencing nausea, vomiting, fatigue, and with some radionuclides (e.g., ${ }^{223} \mathrm{Ra}$ ), hematological suppression. No alteration in endocrine hormone function has been reported, however, these agents may produce reversible bone marrow toxicity in some patients. ${ }^{25}$ The radioisotopes obviously contribute to a patient's overall life-time radiation exposure, and as such, may increase the risk of certain cancers. A key safety concern with these radioisotopes is that they are cleared via renal excretion, and therefore steps need to be taken to prevent reuptake in the kidneys. ${ }^{26}$ To attenuate nephrotoxicity, amino acids (lysine and arginine) and saline are typically infused before and during therapy and patients are asked to void urine frequently. ${ }^{26}$ In addition, antiemetics are typically given prophylactically to prevent nausea and vomiting associated with the amino acid infusion. Due to the potential for increased toxicity, patients with compromised hematological or renal function should avoid RTs.

\section{Theranostic pairs approved or in development}

RT has widespread therapeutic implications beyond NETS and has served as a beacon for the conception of treatments for other cancer types, for example multiple myeloma, leukemia, non-Hodgkin lymphoma, and metastatic castrate-resistant prostate cancer (mCRPC) as well as ovarian and breast cancer (Table 1).,27,28 Prostate specific membrane antigen (PSMA) radioligand therapy is a particularly promising approach to treat advanced-stage prostate cancer. ${ }^{28} \mathrm{PSMA}$ is a transmembrane glycoprotein over expressed in prostate carcinomas and in neovasculature of other solid tumor types. Results in patients with metastatic castration-resistant prostate cancer (mCRPC) have been impressive. A single-arm study of mCRPC patients with progressive disease on second-line hormonal therapy and/or docetaxel chemotherapy showed that $\mathrm{Lu}$ 177-PSMA-617 produced a disease control rates (according to radiographic and molecular responses) of $71 \%$ and $77 \%$ at a median duration of follow up of 28 months, with an overall survival of 14 months and median progression-free survival of 11.8 months. $^{29}$

A case history illustrating the extraordinary response of prostate cancer to another targeted radioligand ${ }^{225}$ Ac-PSMA-617 can be seen in Figure 2. ${ }^{30}$ These images show ${ }^{68} \mathrm{Ga}$-PSMA-11 PET/CT scans of a patient with heavily pretreated $\mathrm{mCRPC}$ with diffuse bone marrow infiltration. The patient was treated with 3 cycles of ${ }^{225}$ Ac-PSMA-617 at bimonthly intervals followed by an additional administration as consolidation therapy. Not only did the patient exhibit virtually complete tumor eradication but also PSA levels declined to less than $0.1 \mathrm{ng} / \mathrm{mL}$. 
Table I Select examples of theranostic pairs approved or in development

\begin{tabular}{|c|c|c|}
\hline Cancer type and molecular target & Imaging & Therapy \\
\hline \multicolumn{3}{|l|}{ Thyroid } \\
\hline Sodium iodide symporter & ${ }^{123}\left|/{ }^{24}\right| /{ }^{131} \mid$ & $\left.|3|\right|^{*}$ \\
\hline \multicolumn{3}{|l|}{ NET } \\
\hline Somatostatin receptor agonists & ${ }^{68} \mathrm{Ga}$ DOTATATE/DOTATOC* & 177Lu DOTATATE* \\
\hline Somatostatin receptor antagonists & ${ }^{68} \mathrm{Ga}-\mathrm{NODAGA}-J \mathrm{R} I \mathrm{I}$ or ${ }^{68} \mathrm{Ga}-\mathrm{OPS} 202$ & ${ }^{177}$ Lu-DOTA-JR II or ${ }^{177}$ Lu-OPS20 I \\
\hline \multicolumn{3}{|l|}{ Prostate cancer } \\
\hline PSMA & ${ }^{68} \mathrm{Ga}$ PSMA-6I7 & ${ }^{177}$ Lu PSMA-6I7 \\
\hline GRP receptor & ${ }^{68} \mathrm{Ga}-\mathrm{NeoBOMBI}$ & ${ }^{177} \mathrm{Lu} \mathrm{NeoBOMBI}$ \\
\hline \multicolumn{3}{|l|}{ Lymphomas, myeloma } \\
\hline CXCR4 & ${ }^{68} \mathrm{Ga}$-pentixafor & ${ }^{177}$ Lu-pentixafor \\
\hline $\mathrm{CD} 20$ & I'In-ibritumomab & ${ }^{90}$ Y-ibritumomab* \\
\hline \multicolumn{3}{|c|}{ Pheochromocytoma and paraganglioma } \\
\hline Norepinephrine transporter & ${ }^{123}$ I-MIBG & ${ }^{|3|}$ I-MIBG \\
\hline \multicolumn{3}{|l|}{ Ovarian, breast } \\
\hline Human EGF & ${ }^{212} \mathrm{~Pb}-\mathrm{TCMC}$-trastuzumab & ${ }^{212} \mathrm{~Pb}-\mathrm{TCMC}$-trastuzumab \\
\hline \multicolumn{3}{|l|}{ Bone metastases } \\
\hline New bone formation & ${ }^{99} \mathrm{Tc}$-oxidronate & ${ }^{153} \mathrm{Sm}$-Lexidronam* \\
\hline Calcimimetic & ${ }^{99} \mathrm{Tc}, 18 \mathrm{NaF}$ & ${ }^{223}$ Ra-dichloride* \\
\hline \multicolumn{3}{|c|}{ Pancreatic ductal adenocarcinoma, colorectal cancer, head and neck cancer } \\
\hline FAPI & ${ }^{68} \mathrm{Ga}-\mathrm{FAPI}-2$ & ${ }^{153} \mathrm{Sm}-\mathrm{FAPI}-2$ \\
\hline
\end{tabular}

For more comprehensive details see Herrmann K, et al. Lancet Oncology 2020; 2 I:el 46-e I56; ${ }^{5}$ Jadvar H, et al. Radiology 20I8; 286: 388-400. ${ }^{*}$ indicates FDA approved

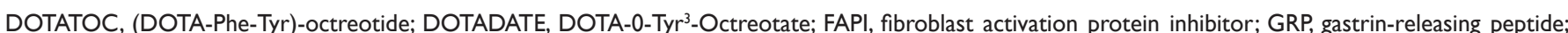
JRII, (Cpa-cyclo(d-Cys-amino-Phe-hydroorotic acid-D-4-amino-Phe(carbamoyl)-Lys-Thr-Cys)-D-Tyr-NH2); MIBG, meta-iodobenzylguanidine; NODAGA (I,4,7-triazacyclononane, I-glutaric acid-4,7-acetic acid); TCMC, 2-(4-isothiocyanotobenzyl)-I, 4, 7, I0-tetraaza-I, 4, 7, I0-tetra-(2-carbamonyl methyl)cyclododecane

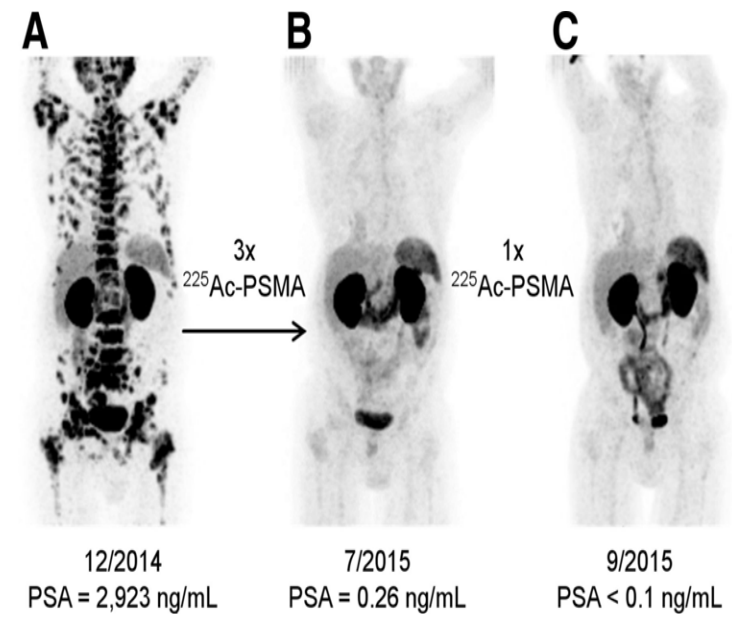

Figure $2{ }^{68} \mathrm{Ga}$-PSMA-II PET/CT scans of patient A. Pretherapeutic tumor spread (A), restaging 2 mo after third cycle of ${ }^{225}$ Ac-PSMA-6I 7 (B), and restaging 2 months after one additional consolidation therapy $(C)$. This research was originally published by C. Kratochwil et al., ${ }^{30} \odot 2016$ by the Society of Nuclear Medicine and Molecular Imaging, Inc (open access).

$\mathrm{PET} / \mathrm{CT}$, positron emission tomography with computed radiographic imaging; PSA, prostate-specific; PSMA, prostate-specific membrane antigen.
Another recent expansion of the theranostic concept involves the C-X-C chemokine receptor type 4(CXCR4). This receptor is present innumerous solid tumors and is related to tumor development and/or metastasis and poor prognosis. ${ }^{6}$ As such, it has garnered interest with respect to chemokine receptor-targeted radiotheranostics. Pentixifor is an antagonist to the CXCR4 receptor, which when coupled to the DOTA chelator, can be labeled with ${ }^{68} \mathrm{Ga}$-pentixifor for imaging and ${ }^{177} \mathrm{Lu}$-pentixifor for therapy and early pilot studies provide encouraging findings in advanced multiple myeloma, small cell lung cancer, and glioblastoma. ${ }^{6,31,32}$

Many human cancers, such as breast cancer, colon cancer, pancreatic cancer, ovarian cancer, and hepatocellular carcinoma over express, albeit it to a modest extent, fibroblast activation protein (FAP), which promotes tumor growth and progression. ${ }^{33,34}$ Normal tissues, on the other hand, show minimal expression of FAP.A proof-of- concept study in mice using a FAP inhibitor revealed ${ }^{64} \mathrm{Cu}-\mathrm{FAPI}-04$ and ${ }^{225} \mathrm{Ac}-$ FAPI-04 may hold promise for use in theranostics to treat pancreatic cancer and possibly other cancer types expressing FAP. ${ }^{33}$

\section{Combination therapies}

Although NETTER-1 showed an impressive lower risk of disease progression or death with ${ }^{177} \mathrm{Lu}$-DOTATATE, only 1 out of 101 patients actually achieved a complete response. ${ }^{18}$ This creates the opportunity 
to co-administer RT with other modalities of cancer treatment. New combination regimens may eventually provide more comprehensive treatment and less morbidity and improved outcomes in the future. A plethora of combinations are currently being evaluated in clinical and preclinical studies with the goal of improving outcomes, minimizing side effects, and overcoming the emergence of resistance. ${ }^{5,35}$

An overview of current and potential therapeutic targets to control proliferation in NETS, in addition to somatostatin receptor radionuclide therapy, is shown in Figure $3 .^{36-38}$ These include targeted therapies such as mammalian target of rapamycin (mTOR) agents (everolimus), inhibition of vascular endothelial growth factor (VEGF) signaling via oral multikinase receptor inhibitor (sunitinib), as well as cytotoxic chemotherapy (streptozotocin and temozolomide). Studies are ongoing to investigate the therapeutic value of combination therapy with these agents. In addition, combining theranostics with external beam radiotherapy may offer increasing tumor radioactivity dose (without overlapping toxicity) and targeting of metastatic disease. ${ }^{35,39}$

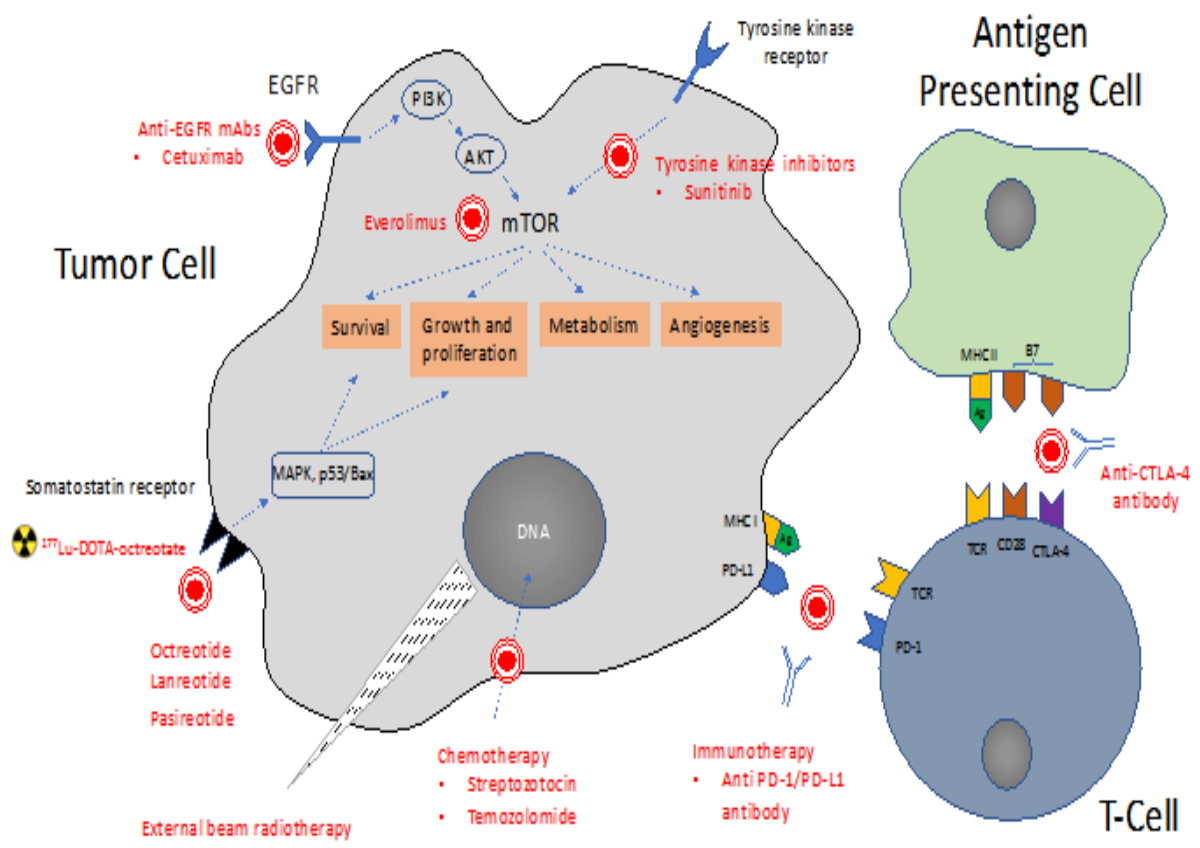

Figure 3 Overview of current and potential therapeutic targets for proliferative control in NET, including octreotide sunitinib, everolimus, temozolomide, and streptotozocin, and immunotherapies, and their respective targets within the neuroendocrine tumor cell. Based in part on Hofland J et al. Endo Rev 2020; 4 I; I33; ${ }^{37}$ Scoville SD et al. Expert Opin Pharmacother. 2020;2I(2):I83-191 ${ }^{38}$ and Weber MM et al. Oncol Res Treat. 20I8;4I:306-3।2.36

$\mathrm{Ag}$, tumor antigen;AKT, serine-threonine kinase; CTLA-4, cytotoxic T-lymphocyte-associated antigen 4; DOTA, I,4,7, I0-tetraaza-cyclododecane-tetraacetic acid; EGFR, epidermal growth factor receptor; mAb, monoclonal antibody; MAPK, mitogen-activated protein kinase; MHC, major histocompatibility complex; mTOR, mammalian target of rapamycin; p53, tumor protein; PD-I, programmed cell death protein I; PD-LI, programmed death-ligand I; PI3K, phosphatidylinositol-3kinase; PRRT, peptide receptor radionuclide therapy;TCR= T-cell receptor.

The prominence of immune surveillance in attenuating neoplastic transformation is well documented. ${ }^{40}$ One of the major mechanisms commandeered by tumors to suppress immune surveillance is the PD-1 (programmed cell death protein 1)/PD-L1 (programmed deathligand 1) pathway. ${ }^{41}$ Tumor cells highjack these suppressive pathways and mitigate antitumor responses in order to develop and progress. ${ }^{41}$ Immune checkpoint inhibitors such as anti PD-1 or anti-CTLA-4 (cytotoxic T-lymphocyte-associated protein 4 ) have proven effective in treating a number of cancer types, including melanoma and lung cancer as well as NETs, particularly Merkel cell carcinoma. ${ }^{36,42}$ These agents appear to be especially promising in patients with progressive or high-grade NECs with microsatellite instability and mutational load. ${ }^{36}$ Clinical trials are underway to study combination therapy with pembrolizumab or nivolumab (IgG4 monoclonal antibodies blocking the PD-1 receptor) and either ${ }^{177} \mathrm{Lu}-\mathrm{PSMA}-617$ in patients with metastatic prostate cancer (NCT03805594) or ${ }^{177} \mathrm{Lu}$-DOTA0Tyr3-Octreotate in small cell lung cancer or advanced or inoperable neuroendocrine tumor of the lung (NCT03325816). These studies should help determine whether combination of RT and immune checkpoint inhibitors offer additional outcome benefits. However, it should be mentioned that anti-PD- 1 and anti-CTLA- 4 antibodies have been associated with endocrinopathies such as hypophysitis, thyroid dysfunction, insulin-deficient diabetes mellitus, and primary adrenal insufficiency) and patients may require hormone replacement and symptom control. ${ }^{43}$

\section{Multidisciplinary approach}

Comprehensive cancer centers around the world are beginning to acknowledge the benefits RT and are adding theranostic nuclear oncology as a core component of their services to cancer patients. ${ }^{28}$ One challenge, however, is the need to consult numerous specialists to manage diverse clinical aspects of NETs and other cancer types. For this reason, a centralized team approach makes sense to improve access to and delivery of care. Some of the key members of this multidisciplinary team include for example: surgeon, endocrinologist, pathologist, radiologist, interventional radiologist, and nuclear medicine physician, and pharmacist. ${ }^{44}$ The overall goals of this team of specialists are enhanced coordination of services, reduced morbidity and mortality, improved patient satisfaction and quality of life, augmented access to evidence-based treatment options, and reduced costs of care. Undoubtedly, this technique will forge a deeper 
collaboration between radiologists and nuclear medicine physicians and it would be prudent for nuclear physicians to gain fluency in the new language of genomics and proteomics. ${ }^{45}$

The world-wide interest in theranostics has prompted Gordon Research Conference, a prestigious non-profit group organizing international scientific conferences on frontier research, to host a Radionucleotide Theranostics for Management of Cancer in July, $2022 .{ }^{46}$ This conference brings together cancer biologists, radiation physicists, receptor pharmacologists, nuclear medicine physicians, and industry representatives with aim of: 1) Identifying new molecular targets and targeting vectors for specific malignancies, 2) Exploring development of novel radionuclides and molecularly targeted radiopharmaceuticals, and 3) Optimizing therapeutic benefits based on an understanding of radionuclide risks to normal tissues.

\section{Conclusion}

Theranostics, the pairing of molecular imaging with receptortargeted therapy, has proven to be a sophisticated, innovative approach to cancer diagnosis, staging, and therapy. The next generation of cancer treatments offers oncologists the ability to treat the specific tumor(s) and offer an individualized therapeutic approach. There are undoubtedly exciting new therapeutic avenues to explore not only in terms of novel receptor agonists/antagonists, isotopes, ligands, chelators, and formulations but also in terms of combination therapies, for example with immune-checkpoint inhibitors and external beam radiation therapy. In addition, other uses are being explored such as intraarterial administration of RTs, targeted radioguided surgery, and tandem treatments, for example, with ${ }^{90}$ Y-DOTATATE and ${ }^{177} \mathrm{Lu}-$ DOTATATE to improve the efficacy without increasing the toxicity.

\section{Funding}

The author received no funding to develop this article.

\section{Acknowledgments}

None.

\section{Conflicts of interest}

The author has no conflicts of interest.

\section{References}

1. Hertz B. A tribute to Saul Hertz: The discovery of the medical uses of radioiodine. World J Nucl Med. 2019;18(1):8-12.

2. Nagarajah J, Janssen M, Hetkamp P, et al. Iodine Symporter Targeting with (124)I/(131)I Theranostics. J Nucl Med. 2017;58(Suppl 2):34S-38S.

3. O'Dorisio TM, Harris AG, O'Dorisio MS. Evolution of neuroendocrine tumor therapy. Surg Oncol Clin N Am. 2020;29(2):145-163.

4. Tornesello AL, Tornesello ML, Buonaguro FM. An overview of bioactive peptides for in vivo imaging and therapy in human diseases. Mini Rev Med Chem. 2017;17(9):758-770.

5. Herrmann K, Schwaiger M, Lewis JS, et al. Radiotheranostics: a roadmap for future development. Lancet Oncol. 2020;21(3):e146-e156.

6. Jadvar H, Chen X, Cai W, et al. Radiotheranostics in cancer diagnosis and management. Radiology. 2018;286(2):388-400.

7. Harris AG, Vinik AI, O'Dorisio TM, et al. Radioligand theranostics in the management of neuroendocrine tumors. Pancreas. 2020;49(5):599-603.

8. Bavelaar BM, Lee BQ, Gill MR, et al. Subcellular targeting of theranostic radionuclides. Front Pharmacol. 2018;9:996.
9. GlaxoSmithKline LLC; Withdrawal of approval of the indication for treatment of patients with relapsed or refractory, low grade, follicular, or transformed CD20 positive non- Hodgkin's lymphoma who have not received prior rituximab; BEXXAR. Federal Register. 2019;78(205):63226-63227.

10. O' Dorisio TM, Redfern JS. Somatostatin and somatostatin-like peptides: Clinical research and clinical applications. Adv Endocrinol Metab. 1990;1:175-230.

11. Redfern JS, O'Dorisio TM. Therapeutic uses of gastrointestinal peptides. Endocrinol Metab Clin North Am.1993;22(4):845-873.

12. Redfern JS, Fortuner WJ, 2nd. Octreotide-associated biliary tract dysfunction and gallstone formation: pathophysiology and management. Am J Gastroenterol. 1995;90(7):1042-1052.

13. Redfern JS, O'Dorisio TM. Gastrointestinal hormones and carcinoid syndrome. In: Felig P, Baxter JD, Frohman LA, eds. Endocrinology and Metabolism. 3rd ed. New York: McGraw-Hill Inc; 1995. 1675-1702 p.

14. Lamberts SWJ, Hofland LJ. Anniversary Review: Octreotide, 40 years later. Eur J Endocrinol. 2019;181(5):R173-R183.

15. Levine R, Krenning EP. Clinical history of the theranostic radionuclide approach to neuroendocrine tumors and other types of cancer: Historical review based on an interview of Eric P. Krenning by Rachel Levine. $J$ Nucl Med. 2017;58(Suppl 2):3S-9S.

16. Krenning EP, Kwekkeboom DJ, Bakker WH, et al. Somatostatin receptor scintigraphy with [111In-DTPA-D-Phe1]- and [123I-Tyr3]-octreotide: the Rotterdam experience with more than 1000 patients. Eur J Nucl Med. 1993;20(8):716-731.

17. Buscombe JR. Evidence base for the use of PRRT. Semin Nucl Med. 2020;50(5):399-404.

18. Strosberg J, El-Haddad G, Wolin E, et al. Phase 3 Trial of 177Lu-Dotatate for midgut neuroendocrine tumors. $N$ Engl J Med. 2017;376(2):125-135.

19. Hennrich U, Kopka K. Lutathera ${ }^{\circledR}$ : The first FDA- and EMA-approved radiopharmaceutical for peptide receptor radionuclide therapy. Pharmaceuticals (Basel). 2019;12(3):114-122.

20. Hennrich U, Benesova M. [(68)Ga]Ga-DOTA-TOC: The First FDA-Approved (68)Ga-Radiopharmaceutical for PET Imaging. Pharmaceuticals (Basel). 2020;13(3):38.

21. Kumar M, Broline S, Amerinia R, et al. Comparison of sensitivity of 68Ga-DOTATATE PET/CT and 111In-Octreotide SPECT in somatostatin positive neuroendocrine tumors. $J$ Nucl Med. 2014;55(Suppl 1):599.

22. Fani M, Nicolas GP, Wild D. Somatostatin receptor antagonists for imaging and therapy. J Nucl Med. 2017;58(Suppl 2):61S-66S.

23. Maxwell JE, Howe JR. Imaging in neuroendocrine tumors: an update for the clinician. Int J Endocr Oncol. 2015;2(2):159-168.

24. Rinzivillo M, Partelli S, Prosperi D, et al. Clinical usefulness of (18) F-Fluorodeoxyglucose positron emission tomography in the diagnostic algorithm of advanced entero-pancreatic neuroendocrine neoplasms. Oncologist. 2018;23(2):186-192.

25. Bodei L, Mueller-Brand J, Baum RP, et al. The joint IAEA, EANM, and SNMMI practical guidance on peptide receptor radionuclide therapy (PRRNT) in neuroendocrine tumours. Eur J Nucl Med Mol Imaging. 2013;40(5):800-816.

26. Hromadik LK, Sturges L. Caring for patients receiving 177Lu-DOTATATE, Lutathera: A treatment of hope for patients with gastroenteropancreatic neuroendocrine tumors. J Radiology Nurs. 2019;38(1):28-32.

27. Tafreshi NK, Doligalski ML, Tichacek CJ, et al. Development of targeted alpha particle therapy for solid tumors. Molecules. 2019;24(23):4314.

28. Turner JH. Recent advances in theranostics and challenges for the future. Br J Radiol. 2018;91(1091):20170893. 
29. Yadav MP, Ballal S, Bal C, et al. Efficacy and safety of 177Lu-PSMA-617 radioligand therapy in metastatic castration-resistant prostate cancer patients. Clin Nucl Med. 2020;45(1):19-31.

30. Kratochwil C, Bruchertseifer F, Giesel FL, et al. 225Ac-PSMA-617 for PSMA-targeted alpha-radiation therapy of metastatic castration-resistant prostate cancer. J Nucl Med. 2016;57(12):1941-1944.

31. Lapa C, Luckerath $\mathrm{K}$, Kleinlein I, et al. 68Ga-Pentixafor-PET/CT for imaging of chemokine receptor 4 expression in glioblastoma. Theranostics. 2016;6(3):428-434.

32. Lapa C, Schreder M, Schirbel A, et al. [(68)Ga]Pentixafor-PET/CT for imaging of chemokine receptor CXCR4 expression in multiple myeloma - Comparison to $[(18) \mathrm{F}] \mathrm{FDG}$ and laboratory values. Theranostics. 2017;7(1):205-212.

33. Watabe T, Liu Y, Kaneda-Nakashima K, et al. Theranostics targeting fibroblast activation protein in the tumor stroma: (64) Cu- and (225)AcLabeled FAPI-04 in pancreatic cancer xenograft mouse models. $J$ Nucl Med. 2020;61(4):563-569.

34. Lindner T, Loktev A, Giesel F, et al. Targeting of activated fibroblasts for imaging and therapy. EJNMMI Radiopharm Chem. 2019;4:16.

35. Gill MR, Falzone N, Du Y, et al. Targeted radionuclide therapy in combined-modality regimens. Lancet Oncol. 2017;18(7):e414-e423.

36. Weber MM, Fottner C. Immune checkpoint inhibitors in the treatment of patients with neuroendocrine neoplasia. Oncol Res Treat. 2018;41(5):306312.

37. Hofland J, Kaltas G, de Herder W. Advances in the diagnosis and management of well-differentiated neuroendocrine neoplasms. Endocr Rev. 2020;41(2):371-403
38. Scoville SD, Cloyd JM, Pawlik TM. New and emerging systemic therapy options for well-differentiated gastroenteropancreatic neuroendocrine tumors. Expert Opin Pharmacother. 2020;21(2):183-191.

39. Dietrich A, Koi L, Zophel K, et al. Improving external beam radiotherapy by combination with internal irradiation. $\mathrm{Br} J$ Radiol. 2015;88(1051):20150042.

40. Disis ML. Immune regulation of cancer. J Clin Oncol. 2010;28(29):45314538 .

41. Skafi N, Fayyad-Kazan M, Badran B. Immunomodulatory role for MicroRNAs: Regulation of PD-1/PD-L1 and CTLA-4 immune checkpoints expression. Gene. 2020;754:144888.

42. Seidel JA, Otsuka A, Kabashima K. Anti-PD-1 and Anti-CTLA-4 therapies in cancer: mechanisms of action, efficacy, and limitations. Front Oncol. 2018;8:86.

43. Chang LS, Barroso-Sousa R, Tolaney SM, et al. Endocrine toxicity of cancer immunotherapy targeting immune checkpoints. Endocr Rev. 2019;40(1):17-65.

44. Metz DC, Choi J, Strosberg J, et al. A rationale for multidisciplinary care in treating neuroendocrine tumours. Curr Opin Endocrinol Diabetes Obes. 2012;19(4):306-313.

45. Turner JH. An introduction to the clinical practice of theranostics in oncology. Br J Radiol. 2018;91(1091):20180440.

46. Radionuclide Theranostics for the Management of Cancer. Gordon Research Conference; 2022. 